\title{
Reticular Oral Lichen Planus
}

\author{
Teresa Cox ${ }^{1,3} \cdot$ Jamie Woodhead $^{2} \cdot$ Brenda L. Nelson $^{1}$
}

Received: 3 October 2018 / Accepted: 26 October 2018 / Published online: 2 November 2018

(c) This is a U.S. Government work and not under copyright protection in the US; foreign copyright protection may apply 2018

\begin{abstract}
Reticular oral lichen planus is a common clinical finding, often found incidentally on routine oral examination. Patients rarely complain of symptoms and the condition does not require treatment, as a result, biopsies and ancillary laboratory evaluation are seldom performed. We present a case of reticular oral lichen planus with a classic clinical presentation and characteristic histologic findings.
\end{abstract}

Keywords Oral lichen planus $\cdot$ Reticular $\cdot$ Lichenoid mucositis $\cdot$ Mucous membrane pemphigoid $\cdot$ Pemphigus vulgaris . Wickham striae

\section{Clinical Features}

A 57 year old male was referred to the hospital dental clinic by his local dentist. The referring dentist requested the bilateral white lesions of the buccal mucosa be evaluated. The patient reported that he was unaware of the lesions and therefore the duration of the process could not be determined. The patient had no symptoms related to these clinical findings, nor any other oral complaints, and denied habitual cheek biting that would cause frictional keratosis. Intraoral examination revealed healthy dentition and periodontium. The exam was notable for fine, white, lace-like lines of the right and left buccal mucosa (Fig. 1). The lesions did not wipe off and were not tender to palpation or manipulation. The remainder of the intraoral findings were unremarkable. A thorough head and neck examination and evaluation of the patient's exposed skin were non-contributory. The patient was given the clinical diagnosis of reticular lichen planus, or Wickham striae. The patient was educated about the condition and his clinical diagnosis was documented. He was, however, concerned about the rendered diagnosis and

Teresa Cox

teresa.m.cox.mil@mail.mil

1 Department of Anatomic Pathology, Naval Medical Center San Diego, San Diego, CA, USA

2 Laboratory Department, Naval Medical Center San Diego, San Diego, CA, USA

3 Department of Pathology, Naval Medical Center San Diego, 34800 Bob Wilson Drive, San Diego, CA 92134-5000, USA requested a biopsy to confirm the diagnosis. The risks and benefits of the procedure were discussed with the patient and a biopsy was performed of the right buccal mucosa.

\section{Histologic Findings}

A biopsy specimen was submitted in $10 \%$ formalin for microscopic evaluation. The tissue sample consisted of a wedge of oral mucosa surfaced by orthokeratinized stratified squamous epithelium. The epithelium was mildly atrophic with blunted rete ridges. At low power, a dense band-like infiltrate of small blue cells is seen in the superficial lamina propria (Fig. 2), which on high power are readily identified as lymphocytes. Focal destruction of the basal cell layer is seen also noting the presence of degenerating keratinocytes, termed Civatte bodies (Fig. 3). No epithelial dysplasia is identified. The diagnosis of lichen planus was rendered.

\section{Discussion}

Lichen planus is a common immune-mediated inflammatory condition which principally affects mucosa and skin. The term lichen derives from the Greek word "leichen", meaning moss-like, while the term "planus", means flat [1]. The name of this condition reflects the similarity the cutaneous lesions have to the scaling algae seen on trees and wooden dwellings. Oral lichen planus is more common than the cutaneous and genital variants, with a prevalence of $1.27 \%$ in American 


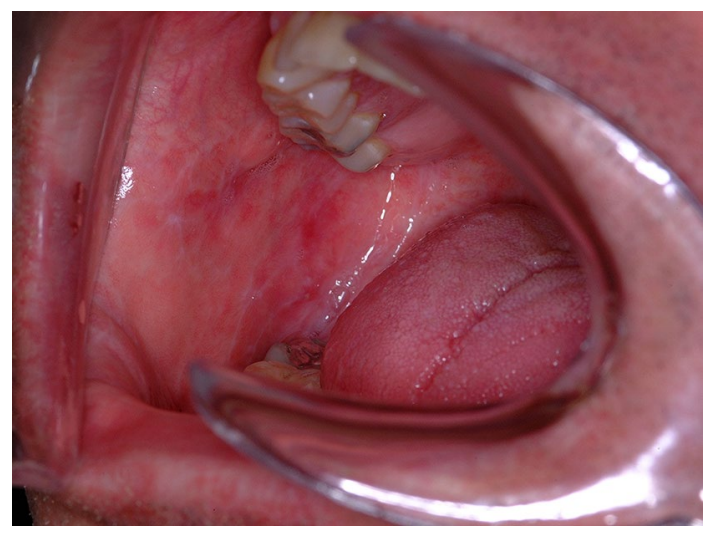

Fig. 1 Intraoral photograph, right buccal mucosa demonstrating fine white lace-like linear lesions characteristic of Wickham striae

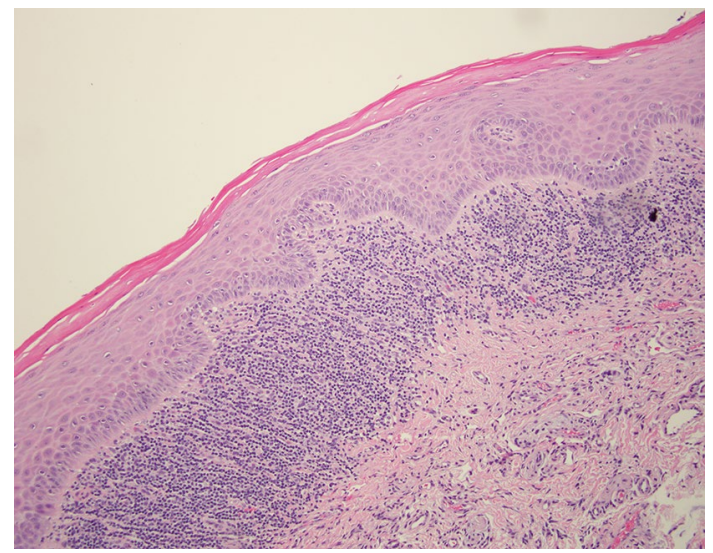

Fig. 2 Low power H\&E photomicrograph shows a mildly atrophic epithelium with a distinct band-like lymphocytic infiltrate in the lamina propria

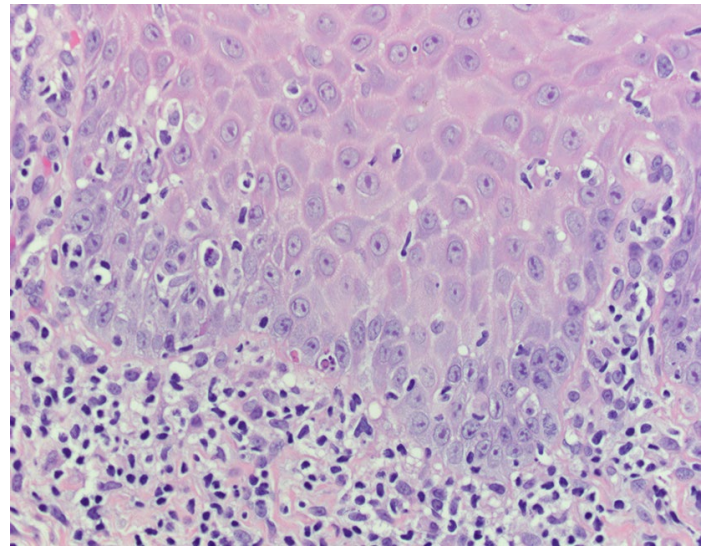

Fig. 3 High power H\&E photomicrograph shows focal degeneration of the basal cells populations. The condition primarily affects adults with a wide age range of $30-80$ years. A slight female predilection (1.5:1) has been reported [2].

The subtypes of OLP include reticular and erosive variants. The reticular variant is the more common of the two and presents with asymptomatic fine, white, linear and lacelike lesions of the buccal mucosa and gingiva referred to as Wickham striae [3]. When the tongue is affected, the presentation is that of flat white plaques. In contrast, the much less common but more clinically significant variant, erosive OLP is symptomatic and is associated with painful ulcerations.

Oral lichen planus is considered to be an idiopathic T-lymphocyte mediated response, involving CD8 + cell activation by $\mathrm{CD} 4+$ lymphocytes, which induces apoptosis of basal keratinocytes [4]. This results in a characteristic histology demonstrating a lymphocytic infiltration of the superficial lamina propria, hyperkeratosis and the presence of Civatte bodies. A saw tooth appearance to the rete ridges is a characteristic histologic finding, however blunted rete ridges and epithelial atrophy may also be present $[4,5]$ as is seen in this particular case. Direct immunofluorescence (DIF), an ancillary study performed on flash-frozen biopsy tissue or fresh biopsy tissue preserved in Michel's solution, is not generally indicated in the evaluation of reticular OLP. However, when performed, DIF demonstrates deposits of fibrinogen along the basement membrane in an irregular granular-like pattern. IgM-positive colloid bodies may also be seen, as well as deposits of $\mathrm{C} 3, \mathrm{IgG}$ and $\operatorname{IgA}$, resulting in a non-specific pattern [6]. This histological presentation, in conjunction with the clinical manifestations of plaques and Wickham striae on the buccal mucosa epitomize the classic presentation of reticular OLP.

While the clinical presentation of reticular OLP may be characteristic, it is important to consider a broad differential diagnosis. A primary consideration is lichenoid mucositis (LM), which may be difficult to distinguish from reticular OLP. Clinically, lichenoid mucositis may also present with fine, white, lace-like and linear lesions on the buccal mucosa that mimic Wickham striae. Additionally, microscopic examination of LM reveals lymphocytic infiltration of the superficial lamina propria, hyperkeratosis, and Civatte body formation remarkably similar to reticular OLP [6]. Likewise, DIF is not specific or diagnostic and may also show irregular granular-like fibrinogen deposits at the basement membrane. Successful differentiation of these two conditions is often dependent upon a complete patient history. The etiology of reticular OLP is currently considered idiopathic, while the etiology of LM may be attributed to numerous extrinsic factors. Dental restorative materials (metals, composite, porcelain and glass ionomers) are contributors and a recent history of a new restoration, or lesions in close association to restorations may be supportive of a diagnosis of LM over OLP. Flavoring agents should also be considered as causation for 
lichenoid mucositis as patients may be sensitive to a number of different products. Mint and tartar-control toothpastes, as well as cinnamon flavorings, are often cited as main offenders. Histologically, the lichenoid reaction to restorative materials may be more robust than seen in reticular OLP with the formation of large lymphoid follicles. The mucositis associated with flavorings, especially cinnamon, may show a mixed inflammatory infiltrate. Neutrophilic exocytosis is also frequently seen [3]. The most significant association with LM is a lichenoid drug reaction. Innumerable medications report LM as a side effect. The major classes of drugs associated with LM include antihypertensives, antibiotics, nonsteroidal anti-inflammatory drugs and antimalarials. Once again, the importance of a thorough medical history is emphasized as the histologic manifestations of a lichenoid drug reaction may resemble that of reticular OLP.

The differential diagnosis of reticular OLP may rarely include splitting diseases such as mucus membrane pemphigoid and pemphigus vulgaris. However, with a proper clinical history (acknowledging the pathologist often does not receive an accurate and complete history), these entities may be quickly ruled out. Microscopically, pemphigoid will demonstrate subepithelial clefting from the lamina propria, with intact basal cells, whereas pemphigus will show a suprabasal cleft. Classically, DIF demonstrates linear deposits of IgG and compliment $\mathrm{C} 3$ at the basement membrane in mucous membrane pemphigoid [7]. These distinctive histologic and DIF patterns are very different from those previously described for reticular OLP.

Depending on the patient's clinical history, chronic graftvs-host disease may also be a consideration. Microscopically the findings may appear non-specific but are characterized by numerous dyskeratotic keratinocytes. Finally, oral dysplasia should be ruled out as it is often the clinical consideration for biopsy. Intense inflammation can make microscopic interpretation difficult, however, epithelial dysplasia will show histologic features not present in reticular OLP such as failure of the squamous epithelium to mature, nuclear pleomorphism, hyperchromasia, and increased mitotic activity [8].

Reticular OLP does not require treatment and does not progress to a more serious disease. If asymptomatic, the diagnosis should be documented in the patient record in conjunction with patient education and reassurance. The provider may want to emphasize that the diagnosis is the reticular variant of OLP, as an internet search of "lichen planus" by the patient may potentially be alarming and cause unnecessary concern. Routine intraoral examination should continue at regular intervals. The clinician should be vigilant for other pathologies so as not to miss a new process that is mistaken for the previously diagnosed reticular oral lichen planus.

Disclaimer The opinions and assertions expressed herein are those of the author and are not to be construed as official or representing the views of the Department of the Navy or the Department of Defense.

Funding This study has no funding.

\section{Compliance with Ethical Standards}

Conflict of interest All the authors declared that they have no conflict of interest.

Ethical Approval This article does not contain any studies with human participants or animals performed by any of the authors.

\section{References}

1. Alrashdan MS, Cirillo N, McCullough M. Oral lichen planus: a literature review and update. Arch Dermatol Res. 2016;308(8):539_ 51. https://doi.org/10.1007/s00403-016-1667-2.

2. Carrozzo M. How common is oral lichen planus? Evid Based Dent. 2008;9(4):112-3. https://doi.org/10.1038/sj.ebd.6400614.

3. Au J, Patel D, Campbell JH. Oral lichen planus. Oral Maxillofac Surg Clin North Am. 2013;25(1):93-100. https://doi. org/10.1016/j.coms.2012.11.007.

4. Gorouhi F, Davari P, Fazel N. Cutaneous and mucosal lichen planus: a comprehensive review of clinical subtypes, risk factors, diagnosis, and prognosis. Sci World J. 2014;2014:742826. https ://doi.org/10.1155/2014/742826.

5. Usatine RP, Tinitigan M. Diagnosis and treatment of lichen planus. Am Fam Physician. 2011;84(1):53-60.

6. Cheng YS, Gould A, Kurago Z, et al. Diagnosis of oral lichen planus: a position paper of the American Academy of Oral and Maxillofacial Pathology. Oral Surg Oral Med Oral Pathol Oral Radiol. 2016;122(3):332-54. https://doi.org/10.1016/j. oooo.2016.05.004.

7. Muller S. Oral lichenoid lesions: distinguishing the benign from the deadly. Mod Pathol. 2017;30:54-67. https://doi.org/10.1038/ modpathol.2016.121.

8. Visser OJ, Kara S, Leidekker M, et al. Isolated oral lichen planus as manifestation of chronic GvHD predicts improved survival after reduced intensity allogeneic stem cell transplantation. Blood. 2006;108(11):5283. 